\title{
Classification of eating disorders: comparison of relative prevalence rates using DSM-IV and DSM-5 criteria
}

Serafino G. Mancuso, J. Richard Newton, Peter Bosanac, Susan L. Rossell, Julian B. Nesci

and David J. Castle

\section{Summary}

DSM-5 contains substantial changes to eating disorder diagnoses. We examined relative prevalence rates of DSM-IV and DSM-5 eating disorder diagnoses using Eating Disorder Examination-Questionnaire diagnostic algorithms in 117 community out-patients. DSM-5 criteria produced a reduction in combined 'other specified feeding or eating disorder' and 'unspecified feeding or eating disorder' diagnoses from $46 \%$ to $29 \%$, an increase in anorexia nervosa diagnoses from $35 \%$ to $47 \%$, the same number of bulimia nervosa diagnoses and a 5\% rate of binge eating disorder diagnoses.

\section{Declaration of interest}

None.

\section{Copyright and usage}

(c) The Royal college of Psychiatrists 2015.
Eating disorders include three relatively distinct illnesses: anorexia nervosa, bulimia nervosa and binge eating disorder. Anorexia and bulimia nervosa were recognised as the two main eating disorders in DSM-IV, ${ }^{1}$ with a third 'eating disorder not otherwise specificed' (EDNOS) diagnosis for eating disorders of clinical significance that did not meet the diagnostic criteria for anorexia or bulimia nervosa. Contained within this EDNOS classification was the provisional diagnosis of binge eating disorder, which is characterised by recurrent binge eating without the inappropriate compensatory behaviours to counteract the effects of binge eating on weight (for example self-induced vomiting, laxative misuse, fasting or overexercise). However, based on the growing evidence that binge eating disorder represented a distinct clinical entity from anorexia and bulimia nervosa, and other forms of EDNOS, ${ }^{2,3}$ binge eating disorder was recognised as a distinct clinical diagnosis in DSM-5. ${ }^{4}$ Other substantial changes have occurred in the diagnosis of eating disorders with DSM-5. ${ }^{4}$ The amenorrhoea criterion has been dropped, and the weight threshold has been marginally increased for anorexia nervosa, and the frequency threshold for binge eating and compensatory behaviours has been lowered for bulimia nervosa. The former DSM-IV EDNOS diagnosis has been replaced by the 'other specified feeding or eating disorder' (OSFED) and 'unspecified feeding or eating disorder' (UFED) diagnoses in DSM-5. The broad OSFED category subsumes five specific exemplars, including atypical anorexia nervosa, subthreshold bulimia nervosa, subthreshold binge eating disorder, purging disorder, and night eating syndrome.

Several studies have compared the relative prevalence rates of eating disorders when DSM-IV diagnoses were reclassified using DSM-5 criteria. Fairburn \& Cooper, ${ }^{5}$ for example, found that OSFED/UFED diagnoses decreased ( $53 \%$ v. 25\%), anorexia nervosa diagnoses increased ( $8 \%$ v. 29\%), and bulimia nervosa diagnoses remained consistent (39\%), with $7 \%$ meeting criteria for binge eating disorder. Sysko et $a l^{6}$ also found that application of the DSM-5 criteria increased anorexia nervosa $(33 \%$ v. $41 \%)$ and bulimia nervosa $(27 \%$ v. $33 \%)$ diagnoses, decreased OSFED/UFED diagnoses (40\% v. 14\%), with 13\% meeting criteria for binge eating disorder. Comparable results were reported by Machado et al, ${ }^{7}$ who found an increase in anorexia (15\% v. 18\%) and bulimia nervosa diagnoses $(12 \%$ v. 15\%), a decrease in OSFED/UFED diagnoses $(73 \%$ v. $51 \%)$, and $6 \%$ fulfilling criteria for binge eating disorder when using the DSM-5 classification. These data suggest that the
DSM-5 criteria may reduce the prevalence of EDNOS by increasing the proportion of individuals who meet diagnostic criteria for a fullthreshold eating disorder, particularly anorexia nervosa and binge eating disorder. Given this apparent reduction in OSFED/UFED diagnoses when applying DSM-5 criteria, we aimed to examine the relative prevalence rates of DSM-IV and DSM-5 eating disorder diagnoses in a large sample of patients with a broad range of diagnoses who presented for treatment in a community out-patient setting.

\section{Method}

Data were collected from 156 participants assessed at the Body Image and Eating Disorder Treatment and Recovery Service (BETRS) of St Vincent's Hospital and Austin Health, Melbourne, Australia between September 2012 and November 2013. Participants were self-, parent- or clinician-referred to the service, which offers out-patient eating disorder treatment. A full description of BETRS can be found elsewhere. ${ }^{8}$ Patients were excluded from analyses if they had missing Eating Disorder Examination-Questionnaire $(\mathrm{EDE}-\mathrm{Q})^{9}$ data that precluded the generation of an EDE-Q diagnosis. As a result of missing data, 39 (25.0\%) were excluded from the current analyses. The 117 participants who were included in the study comprised $113(96.6 \%)$ female and 4 (3.4\%) males. Participants ranged in age from 17 to 54 years (mean 24.8, s.d. $=8.39)$, their body mass index (BMI) ranged from 12.0 to 33.3 (mean 18.2, s.d. =3.47).

The EDE-Q is a 28-item self-report measure that assesses the cognitive and behavioural symptoms of eating disorders. Items that assess the cognitive symptoms of eating disorders are based on a 28-day time frame and are scored on a seven-point Likert scale, ranging from 0 to 6 , with higher scores indicating more severe symptoms. Behavioural symptoms are also based on a 28-day time frame and scores on these items represent the specific frequency of each behaviour. The EDE-Q has demonstrated good psychometric properties and the ability to distinguish between 'cases' and 'non-cases' of eating disorders. ${ }^{10}$ Height and weight were assessed using calibrated instruments. The participant's BMI was calculated by dividing their weight $(\mathrm{kg})$ by the square of their height $(\mathrm{m})$. Menstrual status was based on self-report.

Algorithms were used to classify individuals into one of four diagnostic groups: anorexia nervosa, bulimia nervosa, binge eating 
disorder or EDNOS. Separate diagnostic algorithms were developed for the DSM-IV criteria and the DSM-5 criteria using scores from the EDE-Q. The algorithms were derived from those described by Fairburn \& Cooper $^{5}$ (available from the author on request). However, the algorithms used by Fairburn \& Cooper are based on the clinician-administered Eating Disorder Examination (EDE) but Berg et $a l^{11}$ found that the self-report EDE-Q had a moderate diagnostic concordance with the EDE for DSM-IV and DSM-5 diagnoses ( $\kappa s=0.57$ and 0.60 , respectively).

\section{Results}

Table 1 presents the prevalence of DSM-IV and DSM-5 diagnoses in our sample. Applying the DSM-5 diagnostic criteria caused the prevalence rates of anorexia nervosa to increase by $12.0 \%$ and OSFED/UFED to decrease by $17.1 \%$ when compared with the DSM-IV prevalence rates. Bulimia nervosa prevalence rates were identical across the two diagnostic classifications.

\section{Discussion}

Consistent with previous research, ${ }^{5-7}$ compared with DSM-IV, the DSM- 5 criteria produced a reduction in EDNOS diagnoses from $46 \%$ to $29 \%$ (combined OSFED and UFED diagnoses), an increase in anorexia nervosa from $35 \%$ to $47 \%$, the same number of bulimia nervosa diagnoses and a $5 \%$ rate of binge eating disorder diagnoses. This suggests that more individuals may meet diagnostic criteria for a full-threshold eating disorder when using the DSM-5 criteria, resulting in a reduction of the relative prevalence of EDNOS diagnoses when reclassified as either OSFED or UFED diagnoses. However, our study has limitations. Although the present diagnostic algorithms closely resemble the DSM-IV and DSM-5 criteria, they are not exact replications. First, the EDE-Q does not assess whether there is a disturbance in the manner in which an individual experiences their shape or weight, or whether there is a lack of recognition of the seriousness of low body weight, both of which are included in DSM-IV and DSM-5 criteria for anorexia nervosa. Second, although the DSM-5 criterion A for anorexia nervosa allows consideration of the individual's age, gender, developmental trajectory and physical health when determining low body weight, these factors are not assessed by the EDE-Q. As suggested by Fairburn \& Cooper, ${ }^{5}$ a BMI cut-off of 18.5 was used for the DSM-5 criterion A for anorexia nervosa. Third, because of the time period assessed by the EDE-Q, the duration criteria for binge eating was based on only 28 days. Fourth, DSM-5 criteria B and C for binge eating disorder were not assessed, since the EDE-Q does not contain items that measure the associated features of binge eating disorder or distress regarding binge eating. Additional research is required in which DSM-IV and DSM-5 diagnoses are based on structured clinical interviews, and/or the development of the EDE-Q to include all the relevant items mentioned above. Alternatively, DSM-5 diagnoses could be based on the best available file-review information in a case series previously diagnosed using DSM-IV. Another limitation is that $25 \%$ of available patients had to be excluded because of missing or insufficient data to generate DSM-IV and DSM-5 diagnoses and it remains unclear how these patients may have affected the relative eating disorder prevalence rates. In addition, the sample almost exclusively comprised females seeking treatment in an out-patient service. Therefore, these results may not generalise to males or individuals who are not seeking treatment for their disordered eating.

These limitations notwithstanding, this study examined the classification rates of eating disorders when moving from the
Table 1 Prevalence of DSM-IV and DSM-5 eating disorders in the sample $(n=117)$

DSM-IV, $n(\%) \quad$ DSM-5, $n(\%)$

\begin{tabular}{|lcc|}
\hline Anorexia nervosa & $41(35.0)$ & $55(47.0)$ \\
\hline Bulimia nervosa & $22(18.8)$ & $22(18.8)$ \\
\hline Binge eating disorder & - & $6(5.1)$ \\
\hline Eating disorder not otherwise specified & $54(46.2)$ & - \\
\hline Other specified feeding or eating disorder & - & $30(25.6)$ \\
\hline Unspecified feeding or eating disorder & - & $4(3.4)$ \\
\hline
\end{tabular}

DSM-IV to the DSM-5 criteria. It confirms that the loosened criteria for anorexia nervosa under DSM-5 enable fewer people to be excluded because of their weight or menstrual status. Also a proportion of individuals with a DSM-IV EDNOS diagnosis are subsumed into the new DSM-5 category of binge eating disorder. Yet, a considerable proportion of people with problematic eating are still in the residual OSFED and UFED categories using DSM-5. These individuals deserve more specific research attention if the field is to appreciate the full range of eating disorders.

\section{Serafino G. Mancuso, PhD, St Vincent's Mental Health, Melbourne, Department of Psychiatry, The University of Melbourne, Melbourne and Centre for Excellence in Research Methods, Eastern Hill Academic Centre, Department of Medicine, The Research Methods, Eastern Hill Academic Centre, Department of Medicine, The
University of Melbourne, Melbourne; J. Richard Newton, MBChB, MRCPsych, Department of Psychiatry, The University of Melbourne, Melbourne, Mental Health Clinical Service Unit, Austin Health, Melbourne and Department of Psychological Medicine, Monash University, Melbourne; Peter Bosanac, MD, FRANZCP, St Vincent's Mental Health, Melbourne and Department of Psychiatry, The University of Melbourne, Melbourne; Susan L. Rossell, PhD, Department of Psychiatry, The University of Melbourne, Melbourne and Psychological and Statistical Sciences, Faculty of Life and Social Sciences, Swinburne University of Technology, Melbourne; Julian B. Nesci, DPsych(Clin), St Vincent's Mental Health, Melbourne and Mental Health Clinical Service Unit, Austin Health, Melbourne, Australia; David J. Castle, MD, MRCPsych, St Vincent's Mental Health, Melbourne and Department of Psychiatry, The University of Melbourne, Melbourne, Australia}

Correspondence: Serafino G Mancuso, St Vincent's Mental Health, PO Box 2900, Fitzroy, Melbourne, Australia. Email: sam.mancuso@svha.org.au

First received 15 Dec 2013, final revision 2 Aug 2014, accepted 26 Sep 2014

\section{References}

1 American Psychiatric Association. Diagnostic and Statistical Manual of Mental Disorders (4th edn, revised) (DSM-IV-TR). APA, 2000.

2 Striegel-Moore RH, Franko DL. Should binge eating disorder be included in the DSM-V? A critical review of the state of the evidence. Annu Rev Clin Psychol 2008; 4: 305-24.

3 Wonderlich SA, Gordon KH, Mitchell JE, Crosby RD, Engel SG. The validity and clinical utility of binge eating disorder. Int J Eat Disord 2009; 42: 687-705.

4 American Psychiatric Association. Diagnostic and Statistical Manual of Mental Disorder (5th edn) (DSM-5). APA, 2013.

5 Fairburn CG, Cooper Z. Eating disorders, DSM-5 and clinical reality. Br J Psychiatry 2011; 198: 8-10.

6 Sysko R, Roberto CA, Barnes RD, Grilo CM, Attia E, Walsh BT. Test-retest reliability of the proposed DSM-5 eating disorder diagnostic criteria. Psychiatry Res 2012; 196: 302-8.

7 Machado PPP, Gonçalves S, Hoek HW. DSM-5 reduces the proportion of EDNOS cases: evidence from community samples. Int J Eat Disord 2013; 46 : 60-5.

8 Newton J, Bosanac P, Mancuso S, Castle D. Bridging the gap: does a specialist eating disorder service, aimed at developing a continuum of community care, make a difference? Australas Psychiatry 2013; 21: 365-70.

9 Fairburn CG, Beglin SJ. Assessment of eating disorders: interview or selfreport questionnaire? Int J Eat Disord 1994; 16: 363-70.

10 Berg KC, Peterson CB, Frazier P, Crow SJ. Psychometric evaluation of the Eating Disorder Examination and Eating Disorder Examination-Questionnaire: a systematic review of the literature. Int J Eat Disord 2012; 45: 428-38.

11 Berg KC, Stiles-Shields EC, Swanson SA, Peterson CB, Lebow J, Le Grange D. Diagnostic concordance of the interview and questionnaire versions of the eating disorder examination. Int J Eat Disord 2012; 45: 850-5. 